\title{
Effect of temperature and frequency of dynamic loading in the viscoelastic properties of aluminium alloy 7075-T6
}

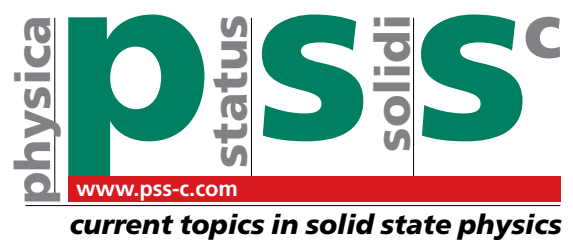

\author{
Jose I. Rojas ${ }^{*}$, Albert Aguiar ${ }^{* *}$, and Daniel Crespo ${ }^{* * *}$ \\ Escola d'Enginyeria de Telecomunicació i Aeroespacial de Castelldefels (EPSC), Universitat Politècnica de Catalunya \\ (UPC Barcelona Tech), Carrer Esteve Terradas 7, 08860 Castelldefels (Barcelona), Spain
}

Received 22 July 2010, revised 28 January 2011, accepted 31 January 2011

Published online 28 June 2011

Keywords viscoelasticity, aluminium alloy, AA 7075, microstructure, phase transformations, GPZ, storage modulus

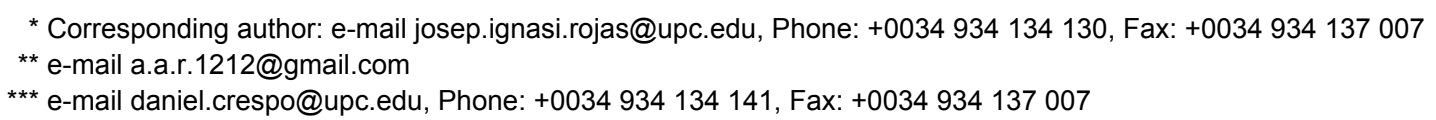

The viscoelastic response of a material offers an alternative method for analyzing its microstructure, phase transformations and fatigue behaviour. In this work, the viscoelastic properties of commercial aluminium alloy (AA) 7075-T6 are studied with a Dynamic-Mechanical Analyzer (DMA), and results are combined with Transmission Electron Microscopy (TEM) and Differential Scanning Calorimetry (DSC) data. In accordance with this analysis, we propose an analytical model for the storage modulus $E^{\prime}$ as a function of temperature, frequency of the dynamic loading and the concentration of GuinierPreston Zones (GPZ). The latter parameter is obtained as a function of temperature after integration and fitting of the model. It is shown that the proposed model fits the experimental data for the storage modulus reasonably well in the prescribed region, and that this fact supports the hypothesis that the change in the storage modulus slope at about $130-160{ }^{\circ} \mathrm{C}$ is due to GPZ decomposition.
1 Introduction The mechanical properties of materials are intimately linked to their microstructure. Thereby, microstructural transformations are reflected on the viscoelastic behaviour [1]. Fatigue, for instance, is a consequence of microstructural changes induced by the viscoelastic response of a material under repeated loading. Accordingly, the characterization of the viscoelastic response of a material offers an alternative method for analyzing its microstructure, phase transformations and fatigue behaviour, aside from enabling a deeper understanding of other essential properties, like damping and yielding [1].

In the present work, the viscoelastic properties of commercial aluminium alloy (AA) 7075-T6 are studied by means of dynamic-mechanical tests, and the results are compared to Transmission Electron Microscopy (TEM) and Differential Scanning Calorimetry (DSC) data available in the literature. AA 7075-T6 features excellent mechanical properties and is suitable to a number of industrial applications, especially in the aerospace and transport industries [2]. This research is aimed at the identification and characterization of the effects of temperature, frequency of dynamic loading, microstructure and phase transformations on its viscoelastic behaviour. Particularly, an analytical model (valid up to $200{ }^{\circ} \mathrm{C}$ ) is proposed for the variation of the viscoelastic response of AA 7075-T6 as a function of temperature, frequency of the dynamic loading and the concentration of Guinier-Preston Zones.

2 Precipitation sequence Age hardening is based on formation of intermetallic products from decomposition of a metastable super-saturated solid solution (SSS), obtained by solution treatment and quenching. The interaction between decomposition products and dislocations is the main responsible for the hardening [3]. AA 7075 belongs to the family of AlZnMg alloys (series 7000). For this family, it is generally accepted that the age-hardening mechanism is essentially based on the following precipitation path [4-6]: $\alpha_{\text {SSS }}$ - GPZI - GPZII - $\eta$ ' - $\eta$; where GPZ are the $\mathrm{Zn} / \mathrm{Mg}$ Guinier-Preston Zones, metastable, coherent clusters precipitated from the supersaturated solid solution, $\eta$ ' is the metastable $\mathrm{MgZn}_{2}$ phase and $\eta$ the equilibrium $\mathrm{MgZn}_{2}$ phase. The particular precipitation path and micro- 
structure, and hence the material properties, depend on the alloy composition and the ageing parameters.

3 Experimental methods A TA Instruments Q800 Dynamic-Mechanical Analyzer (DMA) was used to measure the viscoelastic response of the alloy. The DMA applies a mechanical excitation of selected frequency and amplitude under controlled temperature conditions, and records displacements and stiffness. This allows the evaluation and characterization of intrinsic and extrinsic mechanical properties of the material, namely the viscoelastic behaviour and the creep response. The tested specimens were rectangular plates of $60 \mathrm{~mm}$ in length, 8 to $15 \mathrm{~mm}$ in width and $2 \mathrm{~mm}$ in thickness, which were machine cut from sheet of as-received AA 7075-T6. The mechanical excitation was applied in 3-Point Bending (3PB) mode. The DMA was configured to sequentially apply dynamic loading with frequencies of $100,30,10,3$ and $1 \mathrm{~Hz}$, in isothermal conditions, at different temperatures, from 35 to $375^{\circ} \mathrm{C}$ in step increments of $5^{\circ} \mathrm{C}$. Some tests were set to reach higher temperatures, but results were disregarded as plastic deformations occurred for the applied stress levels. The preload force was $2.0 \mathrm{~N}$ and the applied force $0.5 \mathrm{~N}$. During the tests, data were isothermally recorded and the storage and loss moduli $E$ ' and $E$ " were computed for each of the frequencies at each temperature step.

\section{Results}

4.1 DMA experimental data Figure 1 shows the storage modulus, i.e. the elastic (real) component of the tensile modulus, as a function of temperature for mechanical excitations of frequencies 100, 30, 10, 3 and $1 \mathrm{~Hz}$. As a general trend, the storage modulus decreases initially for all the frequencies. The slope becomes more pronounced at about $130-160{ }^{\circ} \mathrm{C}$, and an inflexion appears around $200-$ $250{ }^{\circ} \mathrm{C}$. Finally, there is a local maximum around $300{ }^{\circ} \mathrm{C}$, after which the storage modulus decreases again. Another important observation is that the storage modulus depends more significantly on frequency at high temperatures, i.e. above $100{ }^{\circ} \mathrm{C}$, being almost insensitive to frequency below this temperature threshold. In addition, at high temperatures, the decrease in the storage modulus at low frequencies is larger than that at higher frequencies.

The loss modulus, which accounts for the energy dissipation during relaxation processes, such as atomic rearrangements or cooperative motion of atomic groups [1], is shown in Fig. 2. It exhibits no significant changes at low temperatures, but starts to increase at about $130-160{ }^{\circ} \mathrm{C}$. This reflects that more energy is dissipated through viscoelastic processes, i.e. internal friction, as temperature increases. It is worth to mention that for all of the tests the loss modulus increases monotonically: no peak is observed in the studied temperature range.

4.2 Modelling of the storage modulus According to available TEM and DSC data in literature [6-10], as well as studies on precipitation sequences and hardening mechanisms [4], it is expected that the mechanical response of the target alloy below $220^{\circ} \mathrm{C}$ is controlled by the presence and decomposition of GPZ. These clusters can be seen in Fig. 3 for an AlCuMg alloy after 2760 min of ageing at room temperature (RT) [3]. Accordingly, we propose an analytical model of the evolution of the storage modulus $E$ ' as a function of temperature $T$, the frequency of the dynamic loading $f$ and the concentration of GPZ, denoted as $C$ :

$$
E^{\prime}(f, T)=E_{0}^{\prime}(f)+E_{1}^{\prime}(f) T+E_{2}^{\prime}(f) C(T)
$$

where $E^{\prime}{ }_{0}$ is the storage modulus at $0 \mathrm{~K}, E^{\prime}{ }_{1}$ accounts for a linear decrease in the storage modulus with temperature due to thermal expansion (see Section 5 for details), and $E^{\prime}{ }_{2}$ reflects the contribution of GPZ to the storage modulus These coefficients are determined by non-linear fit of the model to experimental data. The model is valid when the only significant microstructural transformation taking place is the GPZ dissolution, i.e. up to around $200{ }^{\circ} \mathrm{C}$.

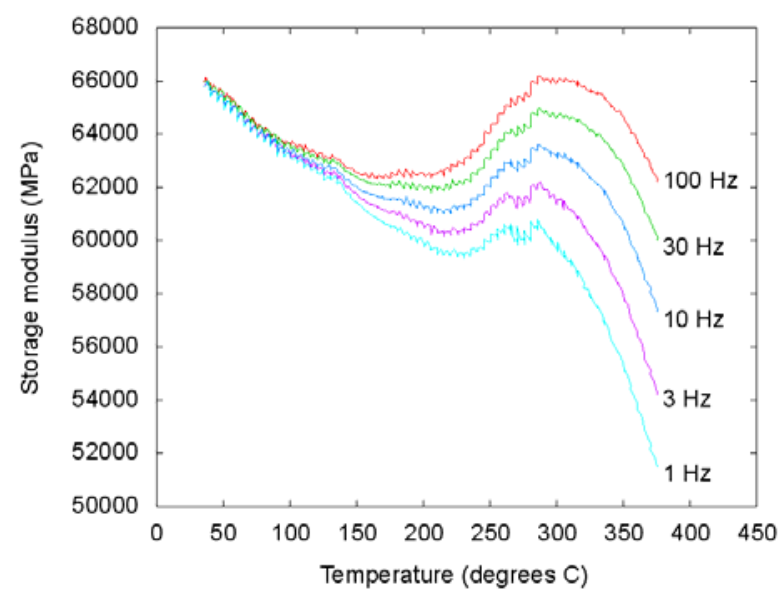

Figure 1 Storage modulus $E$ ' vs. temperature $T$ obtained by dynamic-mechanical tests on AA 7075-T6 at different frequencies.

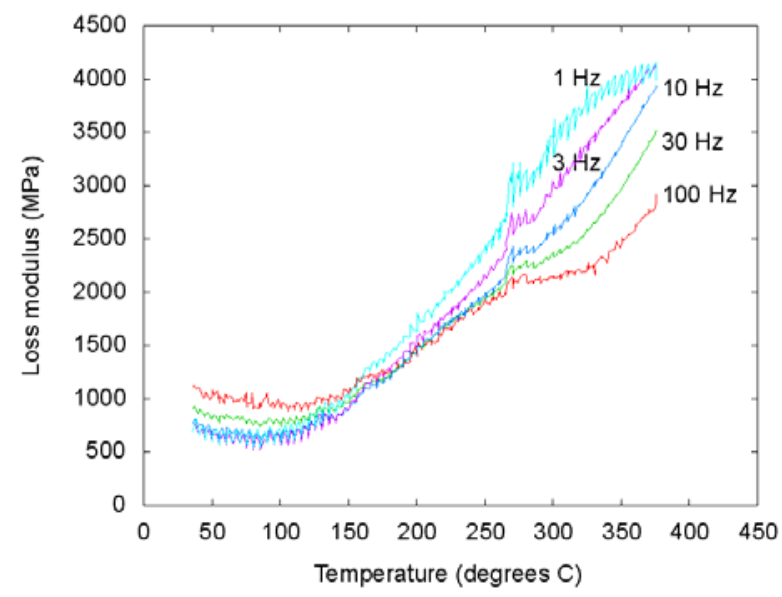

Figure 2 Loss modulus $E$ " vs. temperature $T$ obtained by dynamic-mechanical tests on AA 7075-T6 at different frequencies. 


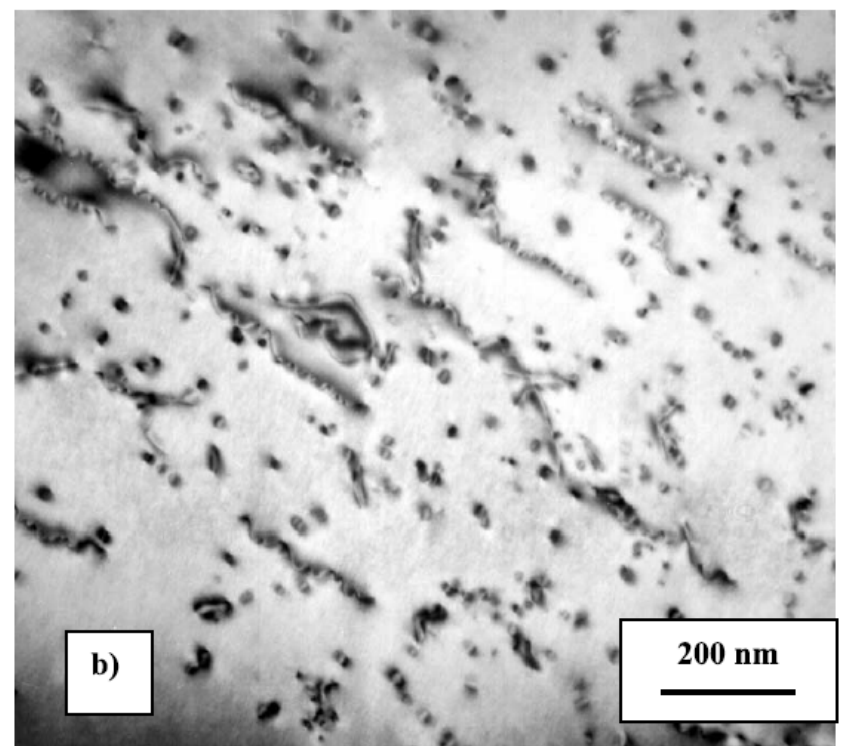

Figure 3 TEM micrograph showing GPZ precipitates in an Al$\mathrm{CuMg}$ alloy after $2760 \mathrm{~min}$ of ageing at room temperature. Reproduced with permission from Abis et al. [3].

Also, being the GPZ dissolution a thermally activated process, the GPZ concentration may follow the Arrhenius behaviour. Thus, as proposed by Jena [11] the transformation rate equation is:

$$
\frac{d C}{d t}=-C k_{0} \exp \left(-\frac{E_{A}}{K T}\right)
$$

where $t$ is the time, $k_{0}$ a pre-exponential coefficient and $E_{A}$ the activation energy of the GPZ dissolution process (both are constants), and $K$ the Boltzmann constant. Alternative expressions to Jena's for modelling GPZ dissolution rate are reported in the literature. Papazian used a 3D diffusion controlled rate expression (Papazian I) and a first order diffusion rate expression (Papazian II) [8]. The latter equations and Eq. (2) were all used and cross-checked to ascertain which one provided a better fit to experimental data.

Numerical integration of the rate equation is performed by using a Runge-Kutta method inside the least-squares non-linear fitting of the storage modulus model to experimental data. This allows us to treat the pre-exponential coefficient and the activation energy as fitting parameters, in addition to coefficients $E^{\prime}{ }_{0}, E^{\prime}{ }_{1}$ and $E_{2}{ }_{2}$. The initial values used for the fitting procedures are directly taken or estimated from data available in the literature, and are summarized in Table 1.

An example of the experimental and numerical storage modulus at $100 \mathrm{~Hz}$, plotted against temperature, is shown in Fig. 4. The computed evolution of GPZ concentration with temperature is shown in Fig. 5, together with the previous numerical values of storage modulus. The GPZ concentration exhibits a characteristic sigmoidal shape.
Table 1 Initial guess parameters for the integration of the transformation rate equation and the non-linear fitting of the proposed storage modulus model, Eq. (2).

\begin{tabular}{lll}
\hline Parameter & Value & References \\
\hline GPZ concentration, $C$ & $4.9 \%$ & {$[5,12-15]$} \\
Pre-exponential coef., $k_{0}$ & $\begin{array}{l}1.0 \times 10^{9} \text { or } \\
1.0 \times 10^{10} \mathrm{~s}^{-1}\end{array}$ & {$[11]$} \\
Activation energy, $E_{A}$ & $1.32 \mathrm{eV} /$ atom & {$[7-10,16,17]$} \\
Coefficient $E^{\prime}{ }_{0}$ & $E^{\prime}$ at RT & - \\
Coefficient $E^{\prime}{ }_{1}$ & $-1.5 \times 10^{7} \mathrm{PaK}^{-1}$ & {$[18]$} \\
Coefficient $E^{\prime}{ }_{2}$ & $5 \times 10^{10} \mathrm{~Pa}^{2}$ & - \\
\hline
\end{tabular}

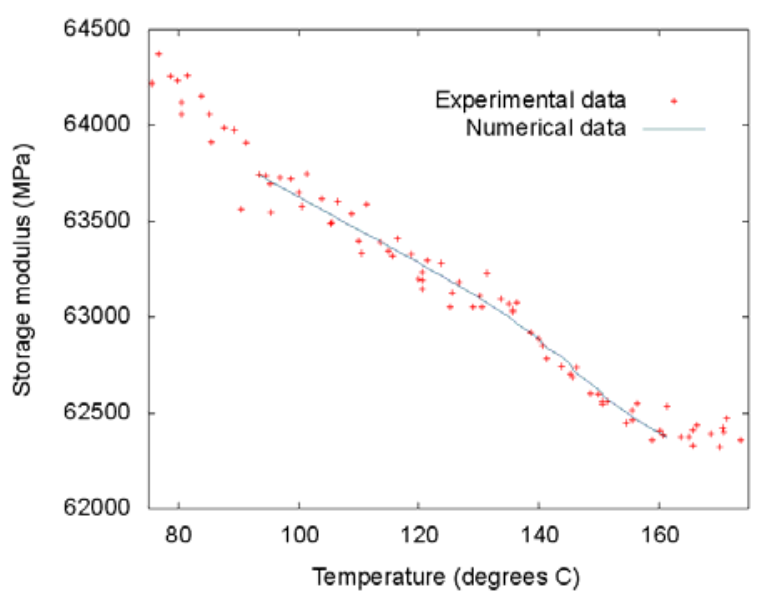

Figure 4 Experimental and numerical results of storage modulus $E^{\prime}$ vs. temperature $T$, for AA 7075-T6, at a frequency of $100 \mathrm{~Hz}$.

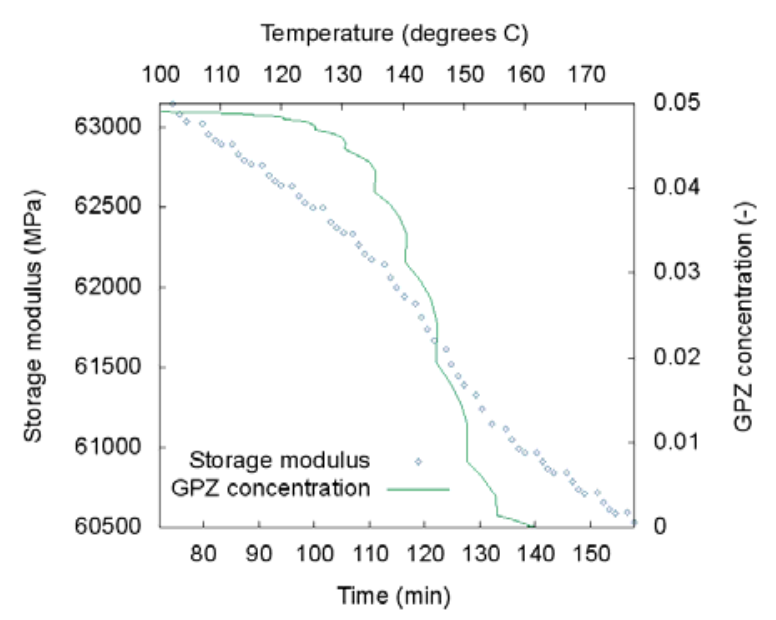

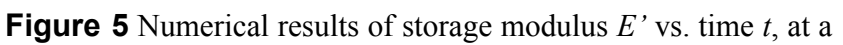
frequency of $100 \mathrm{~Hz}$, and $\mathrm{GPZ}$ concentration $C$ vs. temperature $T$.

The physical quantities of interest, i.e. the preexponential coefficient and activation energy of the GPZ dissolution, for Jena's and Papazian's rate equations are listed in Table 2 . The storage modulus coefficients, $E^{\prime}{ }_{1}$ and $E_{2}{ }_{2}$ are displayed in Fig. 6. 
Table 2 Best-fit values obtained after integration of the considered models. Standard deviations of the fits are given in brackets.

\begin{tabular}{lll}
\hline Model & \multicolumn{2}{l}{ Pre-exponential coefficient, } \\
& $k_{0}\left(\mathrm{~s}^{-1}\right)$ & $E_{A}(\mathrm{eV} /$ atom $)$ \\
\hline Jena & $1.15 \times 10^{9}\left(1.40 \times 10^{9}\right)$ & $1.16(0.08)$ \\
Papazian I & $3.18 \times 10^{9}\left(1.96 \times 10^{9}\right)$ & $1.17(0.10)$ \\
Papazian II & $1.05 \times 10^{9}\left(7.06 \times 10^{7}\right)$ & $1.26(0.15)$ \\
\hline
\end{tabular}

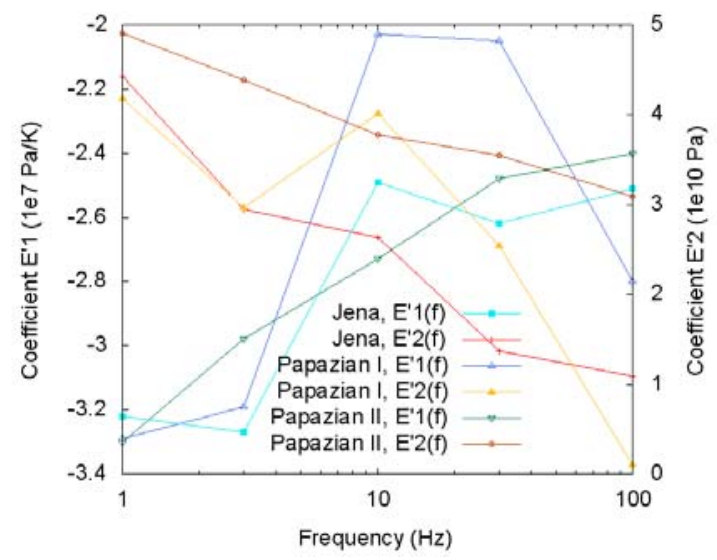

Figure 6 Coefficients $E^{\prime}{ }_{1}$ and $E^{\prime}{ }_{2}$ of the storage modulus model, computed for different types of transformation rate equations.

5 Discussion and conclusions The experimental results confirm that the viscoelastic response depends on temperature and frequency of the mechanical excitation. The initial decrease in the storage modulus can be fairly explained by the dependence of the elastic moduli (elastic stiffness constants) on temperature, which is a well known phenomenon for metals, and particularly aluminium [18]. According to the results in the literature, we assumed a linearly decreasing temperature dependence of the storage modulus, in the studied temperature range. We ascribe the observed change in the storage modulus slope at 130-160 ${ }^{\circ} \mathrm{C}$ to GPZ decomposition, given that GPZ dissolve around these temperatures $[8,11]$, and that the mechanical response of $\mathrm{AlZnMg}$ alloys below $220^{\circ} \mathrm{C}$ is controlled by the presence and decomposition of GPZ [4]. As temperature increases, the relaxation behaviour becomes more prominent, i.e. the storage and loss moduli vary more significantly with the frequency of the mechanical excitation at high temperatures. This phenomenon has also been observed in amorphous alloys [19]. Finally, the fact that the decrease in storage modulus at low frequencies is larger is typically explained by the reduction of the mechanical relaxation time as temperature increases $[2,19]$. At low frequencies, the short relaxation time causes response with large phase lag, which results in a greater decrease of the storage modulus.

We proposed a model for the storage modulus as a function of temperature, frequency of the dynamic loading and GPZ concentration. The model fits the experimental results reasonably well within the prescribed region of validity. In particular, the change in slope at $130-160{ }^{\circ} \mathrm{C}$ is properly accounted for. Hence, we consider that the numerical results support the hypothesis of the variation in the storage modulus slope at $130-160^{\circ} \mathrm{C}$ being caused by GPZ dissolution $[8,11]$. The values of the pre-exponential coefficient and the activation energy of the transformation rate equation for the GPZ dissolution are in good agreement with values reported in the literature by researchers using other techniques $[7-11,16,17]$. This fact confirms the validity of our numerical results for GPZ concentration with temperature, and indicates that the DMA and the proposed approach can be considered a good tool for studying the material microstructure and the kinetics of such phase transformations. Finally, it is noted that the pure Arrhenius dependence proposed by Jena and the 3D diffusion controlled rate expression proposed by Papazian describe similarly the GPZ dissolution process, better than the first order diffusion expression also proposed by Papazian.

Acknowledgements Work supported by the MINCIN grant MAT2007-60087 and the Generalitat de Catalunya grant 2009SGR01251.

\section{References}

[1] A. S. Nowick and B. S. Berry, Anelastic Relaxation in Crystalline Solids (Academic Press, New York, 1972), p. 463.

[2] E. A. Starke and J. T. Staley, Prog. Aerosp. Sci. 32, 161 (1996).

[3] S. Abis, M. Massazza, P. Mengucci, and G. Riontino, Scr. Mater. 45, 686 (2001).

[4] C. E. Macchi, A. Somoza, A. Dupasquier, and I. J. Polmear, Acta Mater. 51, 5151 (2003).

[5] T. Engdahl, V. Hansen, P. J. Warren, and K. Stiller, Mater. Sci. Eng. A 327, 59 (2002).

[6] F. Viana, A. M. P. Pinto, and H. M. C. Santos, J. Mater. Proc. Technol. 93, 54-59 (1999).

[7] U. Batra and S. R. Prabhakar, Trans. Indian Inst. Met. 48, 56 (1995).

[8] J. M. Papazian, Metall. Trans. A 13, 763 (1982).

[9] E. S. Tankins and W. E. Frazier, Mater. Perf. 26, 39 (1987).

[10] S. Yannacopoulos, S. O. Kasap, A. Hedayat, and A. Verma, Can. Metall. Q. 33, 55 (1994).

[11] A. K. Jena, A. K. Gupta, and M. C. Chatuverdi, Acta Metall. 37, 894 (1989).

[12] M. M. Sharma, M. F. Amateaub, and T. J. Eden, J. Alloys Compd. 416, 137 (2006).

[13] J. K. Park and A. J. Ardell, Metall. Trans. A 14, 1959 (1983).

[14] R. Graf, I. J. Polmear, G. Thomas, B. A. Bilby, L. F. Mondolfo, and D.W. Levinson, J. Inst. Met. 86, 535 (1958).

[15] C. E. Lyman and J. B. Vandersande, Metall. Trans. A 7, 1212 (1976).

[16] R. Ferragut, A. Somoza, and A. Dupasquier, J. Phys.: Condens. Matter 8, 8952 (1996).

[17] A. Dupasquier, P. Folegati, A. Rolando, and A. Somoza, Mater. Sci. Forum 175-178, 353 (1995).

[18] J. A. Brammer and C. M. Percival, Exp. Mech. 10, 250 (1970).

[19] H. T. Jeong, E. Fleury, W. T. Kim, D. H. Kim, and K. Hono, J. Phys. Soc. Jpn. 73, 3194 (2004). 